\title{
Dermatology Battles COVID-19 With Comfort
}

\author{
Eric C. Parlette, MD
}

\section{PRACTICE POINTS}

- Be aware of and promote coronavirus disease 2019 guidelines and recommendations from the Centers for Disease Control and Prevention and your local health department.

- Be prepared to push the limits of your comfort zone in an effort to assist the health care community.

W e are in unprecedented times. Severe acute respiratory syndrome coronavirus 2 (SARSCoV-2) is attacking our communities and, as with any battle, we face unexpected challenges from the global pandemic. What can dermatologists, as highly skilled health care experts, do to support the fight against coronavirus disease 2019 (COVID-19)?

In early 2020, I became involved in a fulfilling and stimulating opportunity to contribute as a US Navy reservist, having just returned from a 3-month deployment. I served in the Medical Operations Center aboard the hospital ship USNS Comfort, which was docked in New York Harbor, as liaison to surrounding New York City hospitals. I also served as sole dermatologist on the ship, caring for the dermatologic needs of our team and consulting on numerous COVID-19 inpatients.

In May 2020, upon return to Virginia from New York City, I served as senior medical officer to medically clear other Navy Reserve health care workers returning from the field hospital at the Jacob K. Javits Convention Center of New York and from serving as embedded caregivers in existing New York City hospitals. I share 2 very important observations from my work there: First, COVID-19 is devastatingly real; second, we dermatologists can be valuable team members in the fight against this disease.

It is normal for us to feel scared, confused, and helpless; as $1 \%$ of the physician population, dermatologists represent a small focused fraction of the health care force. Nevertheless, we are all well-trained medical professionals who have taken the same Hippocratic Oath as other physicians. As members of the global health care team, we can each play a role in defeating COVID-19: We can be a trusted voice of reason, set an example, implement safe and effective distancing and hygiene precautions, and assist our local overburdened medical teams.

The magnitude and severity of COVID-19 can create a mass casualty-type phenomenon, overwhelming health care systems if the disease curve is not flattened. We can help flatten that curve by lengthening the pulse duration (to use dermatology jargon): that is, slowing the abrupt impact of cases to allow health care systems to triage, treat, and discharge in a more controlled manner.

\section{How We Can Make a Difference}

Despite representing a fraction of the health care team, we see a larger percentage of the population. On the Comfort, for example, dermatology visits accounted for approximately $20 \%$ of outpatient crew visits. We have an opportunity and a voice to reach a large percentage of the population directly. Whether we are now seeing patients

From River Ridge Dermatology and LewisGale Memorial Hospital Montgomery, Blacksburg, Virginia, and the Naval Leadership and Ethics Center, Newport, Rhode Island.

The author reports no conflict of interest.

The views expressed are those of the author and do not reflect the official policy or position of the US Navy, Department of Defense, or the US Government. Correspondence: Eric C. Parlette, MD, 3706 S Main St, Blacksburg, VA 24060 (ecparlette@yahoo.com).

doi:10.12788/cutis.0134 
face-to-face or virtually, we can spread the public health message and set an example. Wearing masks and social distancing do help to slow and markedly decrease the spread of SARS-CoV-2.

When you see patients in your office, consider the following:

- Have patients wait outside the office in their car and call the receptionist upon arrival.

- Have the receptionist call back the patient when the office is ready.

- Prescreen the patient before having him/her enter the clinic.

- Do not allow handshaking.

- Require everyone to wear a mask.

- Wear gloves.

- Have ample hand sanitizer openly available for all.

- Thoroughly clean or disinfect surfaces between patients.

\section{Recalling the Difficult Experience of a Colleague-Patient}

I think back to a crew member of Comfort who presented with new-onset pruritus and erythematous papules on the arms, legs, and torso. She was an intensive care unit nurse working 13-hour days, every day, for weeks on a COVID-positive unit-double-masked, gowned, wearing eye protection, in a warmer than usual intensive care unit, managing the most critically ill patients she's ever cared for. Outside work, her life consisted of a commute on a government-chartered bus between Comfort and a contracted hotel while eating boxed meals. For 6 hours daily, she would-unsuccessfully-attempt to sleep with raging pruritus. Treating this routine case of eczema had a domino effect, improving her quality of life and thus allowing her to provide better care for the critically ill.

\section{Let Us All Join in the Fight}

As well-educated medical experts, we have the ability and the opportunity to reach outside our comfort zone and assist our medical colleagues. As I saw in New York City, the spectrum of specialists bravely worked together to meet overwhelming demand on the health care system and care for thousands of critically ill and dying patients. Dermatologists treated extensive eczema, ulcers, and other dermatoses on caretakers; triaged patients for appropriate allocation of care; and delivered care outside their comfort zone as physician extenders on inpatient and critical care units.

We are all in this together. I encourage all dermatologists who are in an area of need to ask your health care system how you can join the fight against SARS-CoV- 2 . Let's step forward to help, in recognition of the oath we took to "prevent disease whenever we can." 